\title{
Retraction Note: Remodeling characteristics of slope rock and soil and BIM-based refined management of engineering cost
}

\author{
Jian Xiang ${ }^{1} \cdot$ Yuanyuan Tian $^{2} \cdot$ Jiangbo Shi ${ }^{3}$
}

Published online: 4 January 2022

C) Saudi Society for Geosciences 2022

Retraction Note: Arabian Journal of Geosciences (2021) 14: 950

https://doi.org/10.1007/s12517-021-07243-8

The Editor-in-Chief and the Publisher have retracted this article because the content of this article is nonsensical. The peer review process was not carried out in accordance with the Publisher's peer review policy. Authors Yuanyuan Tian and Jiangbo Shi have not responded to correspondence regarding this retraction. The Publisher has not been able to obtain a current email address for author Jian Xiang.

The original article can be found online at https://doi.org/10.1007/ s12517-021-07243-8.

Jian Xiang

kimi99@aliyun.com

1 School of Civil Engineering, Architecture and Environment, Xihua University, Chengdu 610039, Sichuan, China

2 Institute of Economic Technology, State Grid Leshan Electric Power Supply Company, Leshan 614000, Sichuan, China

3 School of Economics and Management, Leshan Normal University, Leshan 614000, Sichuan, China 